\title{
UK Renal Registry 12th Annual Report (December 2009): Appendix A The UK Renal Registry Statement of Purpose
}

1. Executive summary

2. Introduction

3. Statement of intent

4. Relationships of the UK Renal Registry

5. The role of the UK Renal Registry for patients

6. The role of the UK Renal Registry for nephrologists

7. The role of the UK Renal Registry for Trust managers

8. The role of the UK Renal Registry for commissioning agencies

9. The role of the UK Renal Registry in national quality assurance schemes

10. References and websites

\section{A:1 Executive summary}

1.1 The UK Renal Registry (UKRR) was established by the Renal Association to act as a resource in the development of patient care in renal disease.

1.2 The Registry acts as a source of comparative data for audit, benchmarking, planning, policy and research. The collection and analysis of sequential biochemical and haematological data is a unique feature of the UKRR.

1.3 The Renal Registry Data Set Specification (RRDSS) defines the data items that are required to be sent from participating renal centres for analysis by the UKRR.

1.4 Data is collected quarterly to maintain centre-level quality assurance, with the results being published in an annual report.

1.5 Activity is funded from commissioning agencies by a capitation fee on renal patients.
1.6 The UKRR is responsible, with the express agreement of participants, for providing data to Trusts, Primary Care Trusts (PCTs), commissioning authorities and the European Renal Association European Dialysis and Transplant Association (ERA-EDTA) Registry.

1.7 The development of the Registry is open to influence from all interested parties, including clinicians, Trusts, commissioning authorities and patient groups.

1.8 The Registry is non-profit making and has a registered charitable status through the Renal Association.

\section{A:2 Introduction}

2.1 Registry-based national specialty comparative audit is one of the cornerstones of NHS development. The Renal National Service Framework (NSF), published in two sections in 2004 and 2005 , recommended the participation of all renal centres in comparative audit through the Renal Registry, with co-temporaneous documents defining the necessary information strategies [1-4].

2.2 The shape of future national audit will be set not only by conventional medical criteria, but also by NSF recommendations, prompted through the Healthcare Commission (now renamed as the Healthcare Quality Improvement Partnership). The necessary detail is currently the subject of a formal scoping project, in which the Registry is represented. The final relationship of the Registry

UK Renal Registry, Southmead Hospital, Southmead Road Bristol BS10 5NB, UK

Email: renalreg@renalreg.com 
to the Healthcare Quality Improvement Partnership has yet to be defined.

2.3 The Chief Executives of Trusts are responsible for clinical governance and audit will be an essential part of that agenda [5].

2.4 Demographic information on patients receiving renal replacement therapy (RRT) throughout Europe was collected from 1965 in the Registry of the ERA-EDTA. This voluntary exercise was conducted on paper and by post, demanded considerable effort and time from participating centres and eventually proved impossible to sustain. Latterly, the incompleteness of UK data returns to the ERA-EDTA made it impossible to build a picture of the activity of RRT in the UK for planning and policy purposes. Subsequently, five ad hoc national data collections from England \& Wales were solicited from renal centres in 1992, 1996, 1999, 2002 and 2004 to fill this gap. The UKRR is well placed to put such surveys on a permanent and regular footing and progress towards the inclusion of Chronic Kidney Disease (CKD) is being made.

2.5 Together with the need to know the demographic and structural elements, the NHS has developed a need to underpin clinical activity more rigorously through the scientific evidence base (for example, the Cochrane Initiative) and by quality assurance activity through audit. These initiatives require comprehensive information about the structures, processes and outcomes of RRT, which go well beyond the detail previously compiled by the ERA-EDTA.

2.6 The Registry is recognised as one of the very few high-quality clinical databases available for general use [6]. The collection of data by download of electronic records from routine clinical databases is uncommon, has been highly successful and is being imitated worldwide.

2.7 The Renal Association has made a start in the area of audit by publishing guidelines in 'Renal Standards' documents. It was apparent during the development of the Standards that many of the desirable criteria of clinical performance were uncertain or unknown and that only the accumulated data of practicing renal centres could provide the evidence for advice on best practice and what might be achievable. A common data registration provides the simplest device for such an exercise.
2.8 The continuing emphasis on evidence-based practice is being supported by changes in research funding (Culyer Report and recent national statements), which lean towards collaborative projects and include both basic science and 'health services research' components. It is apparent that an RRT database is invaluable to a wide range of research studies.

2.9 It can be seen that the need for a Registry of RRT has developed for a variety of reasons: international comparisons, national planning, local Trust, PCT and health authority management, standard setting, audit and research. The opportunity for data gathering arises partly from improvements in information technology. Although it was possible to see the need for a national renal database 20 years ago, the circumstances have become ideal for the maintenance of a data repository, supported by the clinical users and resourced for national benchmarking as a routine part of RRT management.

2.10 The provisional expectations of earlier Annual Reports can now be replaced by confident assertions, built on the experience of eleven years of publication, about the role and potential of the UKRR. The integration of the various elements of Renal Association strategy is being pursued through the Clinical Affairs Board (CAB).

\section{A:3 Statement of intent}

The Renal Registry provides a focus for the collection and analysis of standardised data relating to the incidence, clinical management and outcome of renal disease. Data will be accepted quarterly according to the RRDSS by automatic downloading from renal centre databases. There will be a core dataset, with optional elements of special interest that may be entered by agreement for defined periods. A report will be published annually to allow a comparative audit of facilities, patient demographics, quality of care and outcome measures. Participation is mandated through the recommendation in the Renal National Service Framework. There will be an early concentration on RRT, including transplantation, with an extension to other nephrological activity over time. The Registry will provide an independent source of data and analysis on national activity in renal disease. 


\section{A:4 Relationships of the UK Renal Registry}

4.1 The Registry is a registered charity through the Renal Association (No. 2229663). It was established by a committee of the Renal Association, with additional representation from the British Transplantation Society, the British Association for Paediatric Nephrology, the Scottish Renal Registry, Wales and Northern Ireland. There is cross-representation with both the Renal Association Standards and Clinical Trials Committees and the Clinical Affairs Board. The Registry has a Chairman and Honorary Secretary nominated by the Renal Association. The Registry has an observer from the Department of Health, a participant from the National Kidney Federation (NKF) (patients' association), the Royal College of Nursing, the Association for Clinical Biochemistry and a member representing the Health Care Commissioners.

4.2 A number of sub-committees have been instituted as the database and renal centre participation developed, particularly for data analysis and interpretation for the Annual Report. Further specialised panels may be developed for publications and the dissemination of UKRR analyses.

4.3 The Scottish Renal Registry sends data to the UK Renal Registry for joint reporting and comparison.

4.4 The return of English, Welsh and Northern Ireland data to the EDTA-ERA Registry will be through the Renal Registry. The Scottish Renal Registry already sends data directly to the EDTA-ERA Registry.

4.5 A paediatric database has been developed in collaboration with the UKRR, and the two databases are compatible. These two databases are in the process of being integrated, which will allow long-term studies of renal cohorts over a wide age range.

4.6 Close collaboration has been achieved with the NHS Blood and Transplant organisation - (formerly UK Transplant) giving joint benefits. Data aggregation and integration has led to joint presentations and publications. The description of the entire patient journey in RRT by this means is a source of continuing insight and usefulness.

4.7 The basis of participation for renal centres nationally is an agreement to accept the RRDSS for the transmission and retention of data. This is currently increasing to a core dataset of approximately 400 items and further optional elements, which will be returned on a special understanding with the renal centres for a defined period of reporting.
4.8 The UKRR is part of the team undertaking an investigation into the necessary scope of national audit for the Healthcare Quality Improvement Partnership, in the light of the NSF.

4.9 The retention of patient identifiable information, necessary in particular for the adequate tracing of patients, has been approved by the Patient Information Advisory Group (PIAG), under Section 60 of the Health and Social Care Act. This is pending the introduction of mechanisms that will preserve patient anonymity through encryption of a unique patient identifier.

4.10 It is anticipated that the UKRR will receive data from the Secondary Uses Service (SUS) of the national IT programme, Connecting for Health, when it is fully instituted. The detail of data routing from renal centre clinical systems to the national database has yet to be established.

\section{A:5 The role of the UK Renal Registry for patients}

5.1 The goal of the UKRR is to improve care for patients with renal disease. The appropriate use of UKRR information should improve equity of access to care, adequacy of facilities, availability of important but high-cost therapies such as erythrocyte stimulating agents and the efficient use of resources. The continuing comparative audit of the quality of care should facilitate the improvement of care and outcomes of care. It is intended to identify and publish examples of good practice. In such ways, patients will be the ultimate beneficiaries of the exercise.

5.2 A leaflet has been provided, in collaboration with the NKF, by which patients may opt out of the collection of identifiable data by the UKRR if they wish.

5.3 Information from the UKRR will complement the individual records available on 'RenalPatientView' where it is accessible.

\section{A:6 The role of the UK Renal Registry for nephrologists}

6.1 The clinical community have become increasingly aware of the need to define and understand their 
activities, particularly in relation to national standards and in comparison with other renal centres.

6.2 The UKRR is run by a committee of the Renal Association and therefore by colleagues with similar concerns and experience.

6.3 The Renal Standards documents are designed to give a basis for centre structure and performance, as well as patient-based elements such as case mix and outcomes. It is anticipated that Standards will become increasingly based on research evidence and the Cochrane Collaboration has recently resourced reviews of renal topics, which will support this conversion.

6.4 The UKRR data are available to allow the comparative review of many elements of renal centre practice. Centre data are presented to allow a contrast of individual centre activity and results against national aggregated data. Sophisticated analyses of patient survival for example, are a unique resource to exclude any anomalies of performance and standardise for centre caseload, etc.

6.5 Reports of demographic and treatment variables are available to the participating centres for distribution to Trusts, PCTs, Strategic Health Authorities and Commissioners, as well as renal networks, as required and agreed with the centre. Reports should facilitate discussion between clinicians, Trust officers and commissioners.

6.6 Customised data reports can be made available by agreement with the Registry Committee. A charge to cover any costs incurred may be requested.

6.7 The UKRR is developing the publication of focused and extended synopses of chapters from the annual Report. These 'dips' will facilitate the appreciation and application of comparative data and will allow wider distribution.

6.8 The Registry Committee welcome suggestions for topics of national audit or research that colleagues feel are of sufficiently widespread interest for the UKRR to undertake.

6.9 The database has been designed to provide research facilities for future participation in national and international trials. Members of the Renal Association and other interested parties are welcome to apply to the Registry Committee to conduct local or national audit and research using the database. All such projects will need the agreement of the Registry Committee and any costs involved will need to be met by the applicants.
6.10 These facilities will be sustainable only through cooperation between nephrologists and the UKRR. There is a need for high-quality and comprehensive data entry at source.

6.11 The sustaining of data collection, organisation and transmission from peripheral sites is not centrally resourced. The lack of clear status for many informatics staff at centre level, the imminent inroads of the national IT programme Connecting for Health, and the potential disruptions of Agenda for Change will be balanced by the development of formal informatics organisations (The UK Council for Health Informatics Professions (UK CHIP)) [7], the NHS Faculty of Health Informatics [8] and the Association of ICT Professionals in Health and Social Care (ASSIST) [9].

6.12 Centres will need to develop an 'annual informatics plan', to review the maintenance and improvement of data collection organisation and returns to the UKRR. This will help maintain the accuracy, timeliness and completeness of clinical data and also in parallel, support the career development of informatics staff.

\section{A:7 The role of the UK Renal Registry for Trust managers}

7.1 As the basis of the clinical governance initiative, the gathering and presentation of clinical data are regarded as essential parts of routine patient management in the health service.

7.2 One of the principles of health service informatics is that the best data are acquired from clinical information recorded at the point of health care delivery.

7.3 Renal services data entered on local systems by staff directly engaged with patients are likely to be of the highest quality and it is these that the UKRR intends to capture.

7.4 The UKRR provides a cost-effective source of detailed information on renal services.

7.5 The regular reports of the UKRR supply details of patient demographics, treatment numbers, treatment quality and outcomes. Data are compared with both national standards and national performance, for benchmarking and quality assurance. The assessment of contract activity and service delivery is possible through these data returns, 
without the need for further costly Trust or commissioner administrative activity. These data should be particularly valuable to contracts managers and those responsible for clinical governance.

7.6 Data are available on centre case mix, infrastructure and facilities.

7.7 Work is progressing on the data capture and analysis from patients with renal disease other than those requiring RRT and will become available in time (e.g. CKD).

7.8 It is anticipated that Trust interests may be served through the participation of a national Trust representative on the Registry Committee.

\section{A:8 The role of the UK Renal Registry for Commissioners of health care}

8.1 The commissioners of health care include Regional Specialty Commissioning Groups, the networks or joint renal strategy groups supporting them and the Primary Care Trusts.

8.2 The use of information sources such as the UKRR is advised in the National Renal Review in order to promote benchmarking and quality assurance of renal programmes. The comprehensive tracking of relatively small but costly renal cohorts should be regarded as a routine part of speciality case management.

8.3 The UKRR provides validated, comparative reports of renal centre activity on a regular basis to participating centres. These allow assessment of centre performance across a wide range of variables relating to structure, process and outcome measures.

8.4 There are economies of scale in the performance of audit through the UKRR, since multiple local audits are not required.

8.5 The incidence of RRT treated locally, mortality and renal transplant rates should also be of interest. The assessment of referral and treatment patterns of patients with established (end stage) renal failure by postcode analysis indicates the geographical origin. This information also allows the expression of differences relating to geography, ethnicity and social deprivation. These data may also identify potential unmet need in the population and permit assessment on the equity of service provision. In the future, the UKRR database should also provide information on nephrology and pre-dialysis patients (CKD). This will allow a prediction of the need for RRT facilities, as well as indicating the opportunities for beneficial intervention.

8.6 UKRR data are used to track patient acceptance and prevalence rates over time, which allows the modelling of future demand and the validation of these predictions.

8.7 Information on the clinical diagnosis of new and existing RRT patients may help identify areas where possible preventive measures may have maximal effect.

8.8 The higher acceptance rates in the elderly, and the increasing demand from ethnic groups due to a high prevalence of renal, circulatory and diabetic disease, are measurable.

8.9 Comparative data are available in all categories for national and regional benchmarking.

8.10 The UKRR offers independent expertise in the analysis of renal services data and their interpretation, a resource that is widely required but difficult to otherwise obtain.

8.11 The 2009 cost of supporting the UKRR was $£ 18$ per registered patient per annum (2010 £18 per patient), which is less than $0.05 \%$ of the typical cost of a dialysis patient per annum. It is expected that this cost will need to be made explicit within the renal services contract.

8.12 The Registry Committee includes a representative from the health care commissioners. This allows an influence on the development of the UKRR and the topics of interest in data collection and analysis.

\section{A:9 The role of the UK Renal Registry for national quality assurance agencies}

9.1 The role of the UKRR in the national quality assurance programme of the Healthcare Quality Improvement Partnership, (previously the Healthcare Commission) will depend on the decisions on the role and responsibilities of that agency and their means to discharging them.

9.2 The demographic, diagnostic and outcomes data could support the investigation of clinical effectiveness. 
9.3 The case mix information and comorbidity data that would allow better assessment of survival statistics remains incomplete. There is also some clinical scepticism whether 'correction' of outcome data would reflect the realities of clinical practice.
9.4 With the publication of renal centre survival data, consideration of this issue in particular would be welcome in nephrological circles, with correspondence to the Registry Committee (email: renalreg @renalreg.com).

\section{A:10 References}

1 http://www.kidney.org.uk/campaigns/Renal-nsf/pt1-nsf-content-report. pdf [accessed 23.11.05]

2 http://www.kidney.org.uk/campaigns/Renal-nsf/nsf-pt2.pdf [accessed 23.11.05]

3 RNSF IS 1 http://www.dh.gov.uk/assetRoot/04/07/79/25/04077925.pdf

4 RNSF IS $2 \mathrm{http} / / / w w w . d h . g o v . u k / a s s e t R o o t / 04 / 11 / 35 / 05 / 04113505 . p d f$

5 Black N. Clinical governance: fine words or action? $\mathrm{Br}$ Med J 1998; 316:297-8.

6 Black N. High-quality clinical databases: breaking down barriers [Editorial]. Lancet 1999;353:1205-6

7 http://www.ukchip.org.uk/

8 http://www.informatics.nhs.uk/cgi-bin/item.cgi?id=1506 [accessed 23.11.05]

9 http://www.assist.org.uk/ 